\title{
Riemannian corrections to velocity-dependent nuclear forces
}

\author{
Lester Ingber \\ Physical Studies Institute, Drawer W, Solana Beach, California 92075 \\ and Institute for Pure and Applied Physical Sciences, University of California at San Diego, \\ La Jolla, California 92093 \\ (Received 11 April 1983)
}

\begin{abstract}
It is argued that there is a measurable difference between the two-nucleon potential in the Schrödinger wave function and in the potential defined via meson exchanges, when relativistic effects are incorporated as velocity-dependent contributions. This necessitates a reformulation of the usual scattering, deuteron, and nuclear matter calculations.
\end{abstract}

$\left[\begin{array}{c}\text { NUCLEAR STRUCTURE Nuclear matter requires modified velocity-dependent po-- } \\ \text { tential. Nuclear forces, momentum dependence induces Riemannian corrections. }\end{array}\right]$

A current problem in nuclear physics is to understand nuclear matter, i.e., the binding energy and saturation properties. Apart from theoretical considerations of the manybody aspects of this problem, e.g., three-body interactions, there is still much current work being done to improve the nucleon-nucleon potential used as input into calculations of nuclear matter. Basically, the 2- $\pi$ and 3- $\pi$ exchange region and relativistic effects have not yet been definitively established. For example, the Riemannian corrections presented here have not been taken into account, and therefore recent analyses, that conclude that problems in nuclear matter cannot be remedied by a better choice of the two-nucleon potential, may be premature. ${ }^{1}$

As the many-body problem is still essentially only formulated as a nonrelativistic many-body Schrödinger theory, it is necessary that the nucleon-nucleon potential be defined in this context. This requirement has at least two implications: (1) In fitting the parameters of the nucleon-nucleon potential, e.g., coupling constants and masses of exchanged mesons, to scattering data and properties of the deuteron bound state, a Schrödinger representation must be used for these calculations. (For example, a solely dispersion theoretic potential would not suffice to be consistently used for nuclear matter calculations.) (2) Inasmuch as the three basic nuclear physics problems-nucleon-nucleon scattering, nuclear matter, and the deuteron-sensitively test the shape of the entire range of nonrelativistic interactions, the last two physics problems by virtue of cancellations of large contributions, and the first by the wide range of the fit, it is important to include as much physical information regarding meson exchange into the functional form of the potential before the fitting process. That is, the parameters of the potential must be independent of partial waves. The importance of maintaining this integrity of the interaction is increased when extrapolating to new physical phenomena requiring nuclear matter as input, e.g., neutron stars. ${ }^{2}$ Conventionally, the calculation of these basic three nuclear physics problems requires wave functions obtained from

$$
\begin{aligned}
& \left(p^{2} / m+V_{\psi}\right) \psi_{E}=E \psi_{E}, \\
& \left(-\nabla^{2} / m+V_{\psi}\right) \psi_{E}=E \psi_{E}, \\
& \psi_{E}(r)=\phi_{E}(r)+m \int d^{3} r^{\prime} G_{E}\left(r-r^{\prime}\right) V_{\psi}\left(r^{\prime}\right) \psi_{E}\left(r^{\prime}\right),
\end{aligned}
$$

where $\phi_{E}$ is a plane wave for nuclear matter and the scattering problem, and is zero for the bound state. The Green's function $G_{E}$, with energy eigenvalue $E$, is different for each problem. The reduced average nucleon mass is taken to be $m=0.93892 \mathrm{GeV}=0.18528 \mathrm{fm}^{-1}$, using $\bar{h} c=0.197329$ $\mathrm{GeV} \mathrm{fm}$, and setting $\bar{h}=1=c$.

To calculate the properties of nuclear matter, an integral equation is solved of the form

$$
M=V_{M}+\int V_{M} G_{E} M
$$

where $M$ is a partial-wave reduced matrix to accommodate eigenstates of $J^{2}, L^{2}$, and $S^{2}$. For the nuclear matter problem, $M$ is the Brueckner-Goldstone $K$ matrix used to define the energy spectrum; this is derived from a diagrammatic sum of ladder and self-energy $V_{M}$ interactions, and is not simply a Lippman-Schwinger representation of the Schrödinger equation. That representation for the $T$ matrix sometimes is used to calculate the phase shift of the scattering problem, ${ }^{3}$ but often this is accomplished by direct integration of the differential Schrödinger equation. In either case, according to arguments presented here, $V_{\psi}$ should be used for the scattering problem.

The functional form of the potential is determined by setting the Born term, $M=V_{M}$, equal to the sum of Lagrangians of meson-exchange diagrams, ${ }^{3}$ or some corresponding elementary particle representation as referenced below. This is then projected onto five eigenstates of $J^{2}, L^{2}$, and $S^{2}$ complete to order $p^{2} / \mathrm{m}^{2}$, e.g., 1 (central), $\sigma_{1} \cdot \sigma_{2}$ (spinspin), $L \cdot S$ (spin-orbit), $S_{12}=\left(3 \sigma_{1} \cdot \hat{r} \sigma_{2} \cdot \hat{r}-\sigma_{1} \cdot \sigma_{2}\right)$ (tensor), and $\sigma_{1} \cdot p \sigma_{2} \cdot p / m^{2}$. [Note that in Appendix B of Ref. 3 , there is a typographical error in the first sign of the last line; it should read $d \nu(r) / d r$.] Conventionally, $V_{M}$ is used as $V_{\psi}$ in Eq. (1).

The long-ranged spatial extent of the nucleon-nucleon potential is relatively fixed by the low energy scattering and deuteron data, and is functionally determined primarily by the static part of the $\pi$ exchange. The short-ranged force is within the relativistic range of interactions, and given the constraints of the nuclear matter Schrödinger calculations, a soft repulsive core is parametrized with respect to its depth and breadth, although there is certainly room for improvement in this description, e.g., from quark models of highenergy interactions. ${ }^{4-6}$

However, the medium- and high-range scattering data and the nuclear matter calculation are all quite sensitive to the shape of the potential, which is quite steep in the $1 \frac{1}{2}-3 \frac{1}{2}$ 
$\mathrm{GeV}^{-1}$ range. Empirically, the nuclear matter binding energy is $-16 \mathrm{MeV}$ at the Fermi momentum $k_{F}=1.33 \mathrm{fm}^{-1}$, using $k_{F} r_{0}=(9 \pi / 8)^{1 / 3}$, where $r_{0}$ is the saturation point. In this range, momentum-dependent contributions from the nonrelativistic reduction of meson exchanges are important, as pointed out by the first calculations consistently using momentum-dependent forces in all three basic nuclear physics problems. ${ }^{3,7}$ As derived from field theory, contributions to $V_{M}$ of order $p^{2} / m^{2}$ arise from nonrelativistic expansions of nucleon spinors in the Born terms. The use of momentum-dependent potentials is also derived for use in modern potentials, from Regge-pole analyses ${ }^{8}$ and from dispersion theory, especially of the $2-\pi$ and $3-\pi$ exchange region. ${ }^{9}$

For momentum-dependent potentials, previous calculations have used

$$
V\left(r, p^{2}\right)=V\left(r,-\nabla_{r}^{2}\right),
$$

and calculations have demonstrated that the operator dependence can be accommodated in all three physics problems. ${ }^{3}$ Many calculations still use an "effective" potential to simulate the $\nabla$ operator dependence, but this procedure has not yet been explicitly demonstrated to be a good or consistent numerical approximation for the three basic problems.

However, all calculations to date that have used momentum-dependent forces have not considered the effects of the $p^{2}$ dependence in $V\left(r, p^{2}\right)$ on the metric, which is defined by the coefficient of $\nabla^{2}$ in the wave equation, and by the nucleon kinetic energy term in the full Lagrangian in which the meson-exchanged potentials are included as interaction terms.

Studies in other physics specialties have demonstrated that, in a nonflat space, ${ }^{10-14}$ with momentum-dependence affecting only quadratic momentum terms in the Lagrangian of a path-integral defining $\psi$,

$$
V_{\psi}\left(r, p^{2}\right)=V_{M}\left(r, p^{2}\right)+R /(6 m),
$$

where $R$ is the Riemannian curvature scalar. Also, JLS eigenoperators in $\sigma_{1} \cdot p V \sigma_{2} \cdot p$ contain linear $d / d r$ operators. ${ }^{3}$ Similar effects arise in classical statistical mechanics, and in at least one other (bio)physics problem, these Riemannian effects, including contributions from linear momentum terms as well, have been shown to be measurable. ${ }^{15,16}$
These contributions are to be expected if $V_{M}\left(r, p^{2}\right)$ is to be properly correlated with $V_{\psi}\left(r, p^{2}\right)$.

A key point in this argument is that any path-integral-type derivation of the $K$ matrix-i.e., involving the time-folding of a differential propagator-that begins with a two-body momentum-dependent two-nucleon interaction, gives rise to a calculable difference in the effective potential when compared with the potential used in the two-nucleon Schrödinger equation. Since the $K$ matrix itself is not exactly reducible to a Schrödinger partial differential equation, this argument is invoked at the earlier stage first defining the many-body operator containing $V_{M}$, which of course is widely assumed to at least model $V_{\psi}$, before the self-energy and ladder partial sums are taken. In any such derivation, the modification of each kinetic energy term by its momentum-dependent potential defines a nonconstant metric giving rise to this effect.

To specify the momentum operators, first a covariant Schrödinger equation is required for the scalar wave function $\Psi=g^{-1 / 4} \psi$, where $\psi$ is the conventional nonrelativistic wave function. $g=\operatorname{det}\left(g_{i j}\right)$, where $g_{i j}=\left(g^{i j}\right)^{-1}$ is the metric of coordinates $x^{j}$ :

$$
\begin{aligned}
& i \partial_{t} \Psi \equiv H_{\Psi} \Psi=-\frac{1}{2 \mu_{m}}\left(g^{i j} \Psi_{; j}\right)_{; i}+V_{\Psi} \Psi \\
& \equiv\left(-\frac{1}{2 \mu_{m}} g^{-1 / 2} \partial_{i} g^{1 / 2} g^{i j} \partial_{j}+V_{\Psi}\right) \Psi, \\
& \partial_{i}[\cdots]=\partial[\cdots] / \partial x^{i},
\end{aligned}
$$

where $H_{\Psi}$ is the differential Hamiltonian operator, $V_{\Psi}=V_{\Psi}(x)$, and $\mu_{m}=m / 2$ is the reduced average nucleon mass. The path-integral Lagrangian corresponding to $H_{\Psi}$ is

$$
L_{\Psi}=\frac{1}{2 \mu_{m}} g_{i j} \dot{x}^{i} \dot{x}^{j}-V_{\Psi}-R /\left(6 \mu_{m}\right) \text {. }
$$

Note that the covariant divergence of the gradient $\left(g^{i} \Psi_{; j}\right)_{; i}$ reduces to the ordinary Laplacian $\nabla_{x}^{2} \Psi$ in orthogonal coordinates, and that $R$ is defined here to have the same sign as the Gaussian curvature.

$g_{i j}$ is defined here by the effect of $V_{\psi}\left(r ; \nabla_{r}^{2}\right)$ on the flatspace metric $\eta_{i j}$ in $H_{\psi}$. Considering a momentumdependent central potential, e.g., $S$ states,

$$
\begin{aligned}
& i \partial_{t} \psi=H_{\psi} \psi=\left(-\frac{1}{m} g^{-1 / 4} \partial_{i} g^{1 / 2} g^{i j} \partial_{j} g^{-1 / 4}+V_{s}-R /\left(12 \mu_{m}\right)\right) \psi \equiv\left(-\frac{1}{m} \eta^{-1 / 4} \partial_{i} \eta^{1 / 2} \eta^{i j} \partial_{j} \eta^{-1 / 4}+V_{\psi}^{\prime}\right) \psi \equiv\left(\frac{1}{m} p^{2}+V_{\psi}\right) \psi, \\
& g^{i j}=(1+W) \eta^{i j}, \quad \eta=\operatorname{det}\left(\eta_{i j}\right), \\
& V_{\psi}\left(r, p^{2}\right)=V_{S}(r)-R(r) /\left(12 \mu_{m}\right)+\tilde{p}^{2} W(r) / m, \quad \tilde{p}^{2} W=W p^{2}+Y^{i}[W, \eta] \partial_{i}+Z[W, \eta] .
\end{aligned}
$$

This choice of $V_{\psi}$ leaves the path-integral Lagrangian for $\psi$, $L_{\psi}$ (to be associated with $V_{M}$ and the $K$ matrix), $R$ free and "gauge" free: $R$ does not appear in the classical $L_{\psi}$, resulting in $-R /\left(12 \mu_{m}\right)=-R /(6 m)$ in $H_{\psi}$ instead of $-R /\left(6 \mu_{m}\right)$ in $L_{\Psi}$. The covariance of $\left(g^{i j} \Psi_{; j}\right)_{; i}$ dictates the ordering of $p^{2}$ and $W$ in $H_{\psi}$, and also keeps $L_{\psi}$ a quadratic form in $\dot{x}^{i} \dot{x}_{i}$, i.e., not in $\left(\dot{x}^{i}-w^{i}\right)\left(\dot{x}_{i}-w_{i}\right)$ for some nonzero $w^{i}$. Note that $|W|<1$ for all reasonable twonucleon potentials in the nonrelativistic region. In this form, Eq. (6) can be solved straightforwardly, as demonstrated previously. 3,7 Typically,

$$
W(r)=G_{\mu} \frac{\exp (-\mu r)}{m r}
$$

similar to $V_{s}(r) / m$, where $\mu$ is the mass of the meson exchanged, and $G_{\mu}$ is proportional to the square of the nucleon-meson coupling constant, $g_{\mu N N}^{2}$. A proper potential must also be smoothly cut off to blend into the soft core, ${ }^{3}$ but these effects on the shape are not included here. For the $\pi$ meson, the average $\pi$ mass, $\mu_{\pi}=0.13803 \mathrm{GeV}$, yields the longest-ranged force, but $G_{\pi} \sim\left(\mu_{\pi} / m\right)^{2}$, and there is not much contribution to $R$. However, for a scalar 
meson simulating the $\pi-\pi$ exchange, i.e., $\sigma$, or for the $\rho$ meson, the central force has $G \sim 1$, and so these effects might be measurable. As shown in Fig. 3 of Ref. 7, this is the dominant contribution to the ${ }^{1} S_{0}$ state.

To perform this calculation, the arc length in isotropic form is defined as

$$
d s^{2}=(1+W)^{-1}\left(d r^{2}+r^{2} d \theta^{2}+r^{2} \sin ^{2} \theta d \phi^{2}\right) .
$$

This can be put into "standard form," 17 which is more convenient for further calculation:

$$
\begin{aligned}
& d s^{2}=g_{i j} x^{i} x^{j}=A\left(r^{\prime}\right) d r^{2}+r^{\prime 2}\left(d \theta^{2}+\sin ^{2} \theta d \phi^{2}\right), \\
& r^{\prime 2}=[1+W(r)]^{-1} r^{2}, \\
& A\left(r^{\prime}\right)=\left(1-\frac{r}{2} W(r)_{, r}[1+W(r)]^{-1}\right)^{-2}, \\
& {[\cdots]_{, r}=\partial[\cdots] / \partial r .}
\end{aligned}
$$

This gives the Riemann tensor in coordinate space as

$$
\begin{aligned}
& R_{i j k}^{n}=\Gamma_{i j, k}^{n}-\Gamma_{i k, j}^{n}+\Gamma_{i j}^{m} \Gamma_{m k}^{n}-\Gamma_{i k}^{m} \Gamma_{m j}^{n}, \\
& \Gamma_{i j}^{n}=g^{n k}[i j, k] \equiv \frac{1}{2} g^{n k}\left(g_{i k, j}+g_{j k, i}-g_{i j, k}\right),
\end{aligned}
$$

the Ricci tensor as

$$
R_{i j}=R_{i j m}^{m}
$$

and the Riemannian curvature scalar as

$$
R=R_{i}^{i} .
$$

Use of

$$
\begin{aligned}
& R_{r^{\prime}}^{r^{\prime}}=\left(r^{\prime} A^{2}\right)^{-1} A_{, r^{\prime}}, \\
& R_{\theta}^{\theta}=R_{\phi}^{\phi}=r^{\prime-2}+\left(2 r^{\prime} A^{2}\right)^{-1} A_{, r^{\prime}}-\left(r^{\prime 2} A\right)^{-1}, \\
& A_{, r^{\prime}}=A_{, r^{\prime} r^{\prime}}
\end{aligned}
$$

yields

$$
R(r)=\frac{2}{r^{\prime 2}}\left(1-\frac{1}{A\left(r^{\prime}\right)}+\frac{r^{\prime} A\left(r^{\prime}\right)_{, r^{\prime}}}{A\left(r^{\prime}\right)^{2}}\right) .
$$

Although more calculations using the full $V_{\psi}, V_{M}$, and $W \nabla^{2}$ terms are required for definitive analyses, an estimate of the order of Riemannian corrections to nuclear forces is gained by examining graphs of the ratio $R /(6 m): V_{s}$ in Fig. 1(a). For this example, simulating the leading term of a scalar $\sigma$ meson central potential, ${ }^{3} \mu_{\sigma}=0.420$ and $G_{\sigma}=g_{\sigma}^{2}=2.238$,

$$
\begin{aligned}
& V_{s}^{\sigma}=-g_{\sigma}^{2} r^{-1} \exp (-\sigma r), \\
& W^{\sigma} \simeq g_{\sigma}^{2}(2 m r)^{-1} \exp (-\sigma r) .
\end{aligned}
$$

To gauge the sensitivity of this correction to changes in the potential, Fig. 1(b) presents a similar plot, but with $W \rightarrow-W$ set arbitrarily. (Born amplitudes of meson exchanges may contribute with weights of either sign, depending on the eigenvalues of eigenoperators of $J^{2}, L^{2}$, and $S^{2}$, and the isospin of the exchanged meson.)

It now is clear that these Riemannian corrections are small, but not negligible. For example, if a velocitydependent potential were fitted to data using the Schrödinger equation, this potential would have to include the curvature term. The parameters of this fit most likely would be only slightly affected. However, in calculating the nuclear matter $K$ matrix, which is derived from a Lagrangian or Hamiltonian representation, this curvature term would not appear, thereby directly affecting the binding energy and saturation properties. A correction on the order of $1 \%$ in the potential could mean on the order of $1-\mathrm{MeV}$ correction to the binding energy (a cancellation of the kinetic energy and the self-consistent nuclear matter potential), especially if this correction were influential in the steep part of the potential, and if it affected the tensor-to-central ratio of potentials in the ${ }^{3} S_{1} \cdot{ }^{3,7}$

In the absence of a bona fide relativistic field theory, considering nonrelativistically reduced momentum-dependent forces as a perturbation on free nucleon propagators is admittedly a useful but phenomenological procedure. As stressed in Ref. 1, functionally quite different potentials, static and momentum-dependent, give similar results for nuclear matter after they are fit to the scattering and deuteron data, albeit this must yet be tested for momentumdependent potentials correctly including the $\pi-\pi$ region. That is, the binding energy versus saturated $r_{0}$ may lie outside the empirical range. This also might be true for different phenomenologically derived momentum-dependent potentials, in the absence of any distinction between functional contributions used in the scattering/deuteron and nuclear matter calculations. However, in the presence of momentum-dependent forces which surely exist in the considered nonrelativistic range, e.g., those calculated here arise

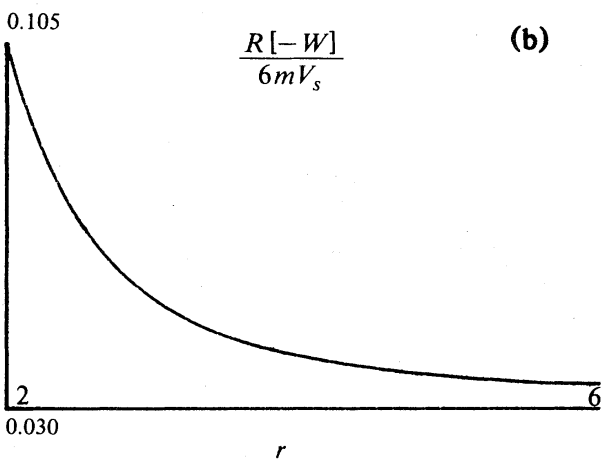

FIG. 1. (a) Using parameters simulating a scalar $\sigma$ central potential, the ratio of $R /(6 \mathrm{~m}): V_{s}$ is plotted vs $r$ in the range 2-6 GeV ${ }^{-1}$. (b) A similar plot to (a), but with $W \rightarrow-W$. For large $r$, this ratio tends asymptotically to $-\frac{1}{6}\left(\mu_{\sigma} / m\right)^{2}=-0.0334$ in (a), and to $+\frac{1}{6}\left(\mu_{\sigma} / m\right)^{2}$ in $(\mathrm{b})$. 
from kinematic factors in the free nucleon spinors (others arise from interaction terms), it is clear that Riemannian corrections account for measureable differences in the potential ultimately used, between calculating fits to the empirical scattering and deuteron data, and calculating properties of nuclear matter.
Construction of plots was facilitated with the PDP-10 MACSYMA Consortium algebraic manipulator at the Massachusetts Institute of Technology, supported in part by U.S. Energy Research and Development Administration Grant No. E(11-1)-3070 and National Aeronautics and Space Administration Grant No. NSG 1323.
${ }^{1}$ B. D. Day, Phys. Rev. Lett. 47, 226 (1981).

2J. -R. Buchler and L. Ingber, Nucl. Phys. A170, 1 (1971).

${ }^{3}$ L. Ingber, Phys. Rev. 174, 1250 (1968).

${ }^{4}$ W. R. Frazer, L. Ingber, C. H. Mehta, C. H. Poon, D. Silverman, K. Stowe, P. D. Tin, and H. J. Yesian, Rev. Mod. Phys. 44, 284 (1972).

${ }^{5}$ Y. Suzuki and K. T. Hecht, Phys. Rev. C 27, 299 (1983).

${ }^{6}$ H. Toki, Z. Phys. A 294, 173 (1980).

${ }^{7}$ L. Ingber and R. M. Potenza, Phys. Rev. C 1,112 (1970).

${ }^{8}$ M. M. Nagels, T. A. Rijken, and J. J. de Swart, Phys. Rev. D $\underline{17}$, 768 (1978).
${ }^{9}$ M. Lacombe, B. Loiseau, J. M. Richard, R. Vinh Mau, J. Coté, P. Pirès, and R. de Tourreil, Phys. Rev. C 21, 861 (1980).

${ }^{10}$ K. S. Cheng, J. Math. Phys. 13,1723 (1972).

${ }^{11}$ H. Dekker, Phys. Rev. A 19, 2102 (1979).

${ }^{12}$ B. S. DeWitt, Rev. Mod. Phys. 29, 377 (1957).

${ }^{13}$ R. Graham, Z. Phys. B 26, 397 (1977).

${ }^{14} \mathrm{~F}$. Langouche, D. Roekaerts, and E. Tirapegui, J. Phys. A $\underline{13}, 449$ (1980).

${ }^{15}$ L. Ingber, Physica D $\underline{5}, 83$ (1982).

${ }^{16}$ L. Ingber, Phys. Rev. A 28,395 (1983).

${ }^{17}$ S. Weinberg, Gravitational Cosmology (Wiley, New York, 1972). 